\title{
Existence and Uniqueness Results for a Coupled System of Nonlinear Fractional Differential Equations with Antiperiodic Boundary Conditions
}

\author{
Huina Zhang ${ }^{1,2}$ and Wenjie Gao ${ }^{1}$ \\ ${ }^{1}$ Institute of Mathematics, Jilin University, Changchun 130012, China \\ ${ }^{2}$ College of Science, China University of Petroleum (East China), Qingdao 266555, China \\ Correspondence should be addressed to Wenjie Gao; wjgao@mail.jlu.edu.cn
}

Received 28 October 2013; Accepted 13 December 2013; Published 12 January 2014

Academic Editor: Douglas Anderson

Copyright (C) $2014 \mathrm{H}$. Zhang and W. Gao. This is an open access article distributed under the Creative Commons Attribution License, which permits unrestricted use, distribution, and reproduction in any medium, provided the original work is properly cited.

This paper studies the existence and uniqueness of solutions for a coupled system of nonlinear fractional differential equations of order $\alpha, \beta \in(4,5]$ with antiperiodic boundary conditions. Our results are based on the nonlinear alternative of Leray-Schauder type and the contraction mapping principle. Two illustrative examples are also presented.

\section{Introduction}

In this paper, we consider the existence and uniqueness of solutions for the following coupled system of nonlinear fractional differential equations:

$$
\begin{gathered}
{ }^{c} D^{\alpha} x(t)+f(t, x(t), y(t))=0, \quad t \in[0, T], \\
{ }^{c} D^{\beta} y(t)+g(t, x(t), y(t))=0, \quad t \in[0, T], \\
x^{(i)}(0)=-x^{(i)}(T), \quad i=0,1,2,3,4, \\
y^{(i)}(0)=-y^{(i)}(T), \quad i=0,1,2,3,4,
\end{gathered}
$$

where $4<\alpha, \beta \leq 5$, and ${ }^{c} D^{\alpha}$ denotes the Caputo fractional derivative of order $\alpha$. Here our nonlinearity $f, g:[0, T] \times \mathbb{R} \times$ $\mathbb{R} \rightarrow \mathbb{R}$ are given continuous functions.

Fractional differential equations have recently been addressed by many researchers in various fields of science and engineering, such as rheology, porous media, fluid flows, chemical physics, and many other branches of science; see [1-4]. As a matter of fact, fractional-order models become more realistic and practical than the classical integer-order models; as a consequence, there are a large number of papers and books dealing with the existence and uniqueness of solutions to nonlinear fractional differential equations; see [5-14]. The study of a coupled system of fractional order is also very significant because this kind of system can often occur in applications; see [15-17].

Antiperiodic boundary value problems arise in the mathematical modeling of a variety of physical process; many authors have paid much attention to such problems; for examples and details of Antiperiodic boundary conditions, see [5, 18-22]. In [5], Alsaedi et al. study an Antiperiodic boundary value problem of nonlinear fractional differential equations of order $q \in(4,5]$.

It should be noted that, in [23], Ntouyas and Obaid have researched a coupled system of fractional differential equations with nonlocal integral boundary conditions, but this paper researches a coupled system of fractional differential equations with Antiperiodic boundary conditions. On the other hand, in $[5,19]$, the authors have discussed some existence results of solutions for Antiperiodic boundary value problems of fractional differential equation but not the coupled system. The rest of the papers above for the coupled systems have been devoted to the case of 
Riemann-Liouville fractional derivatives but not the Caputo fractional derivatives.

This paper is organized as follows. In Section 2, we recall some basic definitions and preliminary results. In Section 3, we give the existence results of (1) by means of the Leray-Schauder alternative; then we obtain the uniqueness of solutions for system (1) by the contraction mapping principle. We give two examples in Section 4 to illustrate the applicability of our results.

\section{Background Materials}

For the convenience of the readers, we present here some necessary definitions and lemmas which are used throughout this paper.

Definition 1 (see $[2,3]$ ). The Riemann-Liouville fractional integral of order $\alpha>0$ of a function $y:(0, \infty) \rightarrow \mathbb{R}$ is given by

$$
I^{\alpha} y(t)=\frac{1}{\Gamma(\alpha)} \int_{0}^{t}(t-s)^{\alpha-1} y(s) d s
$$

provided the right hand side is pointwise defined on $(0, \infty)$.

Definition 2 (see $[2,3]$ ). The Caputo fractional derivative of order $\alpha>0$ of a continuous function $y:(0, \infty) \rightarrow \mathbb{R}$ is given by

$$
{ }^{c} D^{\alpha} y(t)=\frac{1}{\Gamma(n-\alpha)} \int_{0}^{t} \frac{y^{(n)}(s)}{(t-s)^{\alpha-n+1}} d s
$$

where $n=[\alpha]+1$ and $[\alpha]$ denotes the integer part of number $\alpha$, provided that the right side is pointwise defined on $(0, \infty)$.

Lemma 3 (see [19]). Consider $I^{\alpha c} D^{\alpha} x(t)=x(t)+C_{0}+C_{1} t+$ $C_{2} t^{2}+\cdots+C_{n-1} t^{n-1}$, for some $c_{i} \in \mathbb{R}, i=0,1,2, \ldots, n-1$, where $n=[\alpha]+1$ and $[\alpha]$ denotes the integer part of number $\alpha$.

Lemma 4 (see [5]). For any $y \in C[0, T]$, the unique solution of the boundary value problem

$$
\begin{gathered}
{ }^{c} D^{q} x(t)=y(t), \quad t \in[0, T], 4<q \leq 5, \\
x^{(i)}(0)=-x^{(i)}(T), \quad i=0,1,2,3,4
\end{gathered}
$$

is

$$
x(t)=\int_{0}^{T} G(t, s) y(s) d s
$$

where $G(t, s)$ is the Green function given by

$G(t, s)$

$$
=\left\{\begin{array}{l}
\frac{2(t-s)^{q-1}-(T-s)^{q-1}}{2 \Gamma(q)}+\frac{(T-2 t)(T-s)^{q-2}}{4 \Gamma(q-1)} \\
+\frac{t(T-t)(T-s)^{q-3}}{4 \Gamma(q-2)}+\frac{\left(6 T t^{2}-4 t^{3}-T^{3}\right)(T-s)^{q-4}}{48 \Gamma(q-3)} \\
+\frac{\left(2 T t^{3}-t T^{3}-t^{4}\right)(T-s)^{q-5}}{48 \Gamma(q-4)}, \quad 0<s<t<T, \\
-\frac{(T-s)^{q-1}+\frac{(T-2 t)(T-s)^{q-2}}{4 \Gamma(q)}+\frac{(q-1)}{\left(6 T t^{2}-4 t^{3}-T^{3}\right)(T-s)^{q-4}}}{+\frac{t(T-t)(T-s)^{q-3}}{4 \Gamma(q-2)}+\frac{48 \Gamma(q-3)}{48 \Gamma(q-4)},} \quad 0<t<s<T . \\
+\frac{\left(2 T t^{3}-t T^{3}-t^{4}\right)(T-s)^{q-5}}{}
\end{array}\right.
$$

Let

$G_{1}(t, s)$

$$
=\left\{\begin{array}{l}
\frac{(t-s)^{\alpha-1}-(1 / 2)(T-s)^{\alpha-1}}{\Gamma(\alpha)}+\frac{(T-2 t)(T-s)^{\alpha-2}}{4 \Gamma(\alpha-1)} \\
+\frac{t(T-t)(T-s)^{\alpha-3}}{4 \Gamma(\alpha-2)}+\frac{\left(6 T t^{2}-4 t^{3}-T^{3}\right)(T-s)^{\alpha-4}}{48 \Gamma(\alpha-3)} \\
+\frac{\left(2 T t^{3}-t T^{3}-t^{4}\right)(T-s)^{\alpha-5},}{48 \Gamma(\alpha-4)} 0<s<t<T, \\
-\frac{1}{2 \Gamma(\alpha)}(T-s)^{\alpha-1}+\frac{(T-2 t)(T-s)^{\alpha-2}}{4 \Gamma(\alpha-1)} \\
+\frac{t(T-t)(T-s)^{\alpha-3}}{4 \Gamma(\alpha-2)}+\frac{\left(6 T t^{2}-4 t^{3}-T^{3}\right)(T-s)^{\alpha-4}}{48 \Gamma(\alpha-4)}, \quad 0<t<s<T,
\end{array}\right.
$$

$G_{2}(t, s)$

$$
=\left\{\begin{array}{l}
\frac{(t-s)^{\beta-1}-(1 / 2)(T-s)^{\beta-1}}{\Gamma(\beta)}+\frac{(T-2 t)(T-s)^{\beta-2}}{4 \Gamma(\beta-1)} \\
+\frac{t(T-t)(T-s)^{\beta-3}}{4 \Gamma(\beta-2)}+\frac{\left(6 T t^{2}-4 t^{3}-T^{3}\right)(T-s)^{\beta-4}}{48 \Gamma(\beta-3)} \\
+\frac{\left(2 T t^{3}-t T^{3}-t^{4}\right)(T-s)^{\beta-5}}{48 \Gamma(\beta-4)}, 0<s<t<T, \\
-\frac{1}{2 \Gamma(\beta)}(T-s)^{\beta-1}+\frac{(T-2 t)(T-s)^{\beta-2}}{4 \Gamma(\beta-1)} \\
+\frac{t(T-t)(T-s)^{\beta-3}}{4 \Gamma(\beta-2)}+\frac{\left(6 T t^{2}-4 t^{3}-T^{3}\right)(T-s)^{\beta-4}}{48 \Gamma(\beta-3)} \\
+\frac{\left(2 T t^{3}-t T^{3}-t^{4}\right)(T-s)^{\beta-5}}{48 \Gamma(\beta-4)} \quad 0<t<s<T .
\end{array}\right.
$$

We call $\left(G_{1}, G_{2}\right)$ Green's function for problem (1).

We define the space $X=\{x(t) \mid x(t) \in C[0, T]\}$ endowed with $\|x\|_{X}=\max _{t \in[0, T]}|x(t)|$; for $(x, y) \in X \times X$, 
let $\|(x, y)\|_{X \times X}=\|x\|_{X}+\|y\|_{X}$. Obviously, $\left(X,\|\cdot\|_{X}\right)$ is a Banach space, and the product space $\left(X \times X,\|(\cdot, \cdot)\|_{X \times X}\right)$ is also a Banach space.

Consider the following coupled system of the integral equations:

$$
\begin{aligned}
& x(t)=\int_{0}^{T} G_{1}(t, s) f(s, x(s), y(s)) d s, \\
& y(t)=\int_{0}^{T} G_{2}(t, s) g(s, x(s), y(s)) d s .
\end{aligned}
$$

As a result, differential problem (1) turns into integral problem (8), and here is a conclusion about the relationship between their solutions.

Lemma 5. Assume that $f, g:[0, T] \times \mathbb{R} \times \mathbb{R} \rightarrow \mathbb{R}$ are continuous functions. Then $(x, y) \in(X, X)$ is a solution of $(1)$ if and only if $(x, y) \in(X, X)$ is a solution of system (8).

Proof. The proof is immediate from the discussion above, and we omit the details here.

Let $F: X \times X \rightarrow X \times X$ be an operator defined as $F(x, y)(t)=\left(F_{1}(x, y)(t), F_{2}(x, y)(t)\right)$, where

$$
\begin{aligned}
& F_{1}(x, y)(t)=\int_{0}^{T} G_{1}(t, s) f(s, x(s), y(s)) d s, \\
& F_{2}(x, y)(t)=\int_{0}^{T} G_{2}(t, s) g(s, x(s), y(s)) d s .
\end{aligned}
$$

It is obvious that a fixed point of the operator $F$ is a solution of problem (1).

\section{Main Results}

In this section, we will discuss the existence and uniqueness of solutions for problem (1).

Lemma 6. One can conclude that the Green functions $G_{1}(t, s)$, $G_{2}(t, s)$ satisfy the following estimates:

$$
\begin{aligned}
& \int_{0}^{T}\left|G_{1}(t, s)\right| d s \\
& \leq \frac{T^{\alpha}}{\Gamma(\alpha+1)}\left(\frac{3}{2}+\frac{5 \alpha^{4}-14 \alpha^{3}+55 \alpha^{2}+146 \alpha}{768}\right)=U_{1}, \\
& t \in[0, T],
\end{aligned}
$$

$$
\begin{array}{r}
\int_{0}^{T}\left|G_{2}(t, s)\right| d s \\
\leq \frac{T^{\beta}}{\Gamma(\beta+1)}\left(\frac{3}{2}+\frac{5 \beta^{4}-14 \beta^{3}+55 \beta^{2}+146 \beta}{768}\right)=U_{2}, \\
t \in[0, T],
\end{array}
$$

$$
\begin{aligned}
\int_{0}^{T}\left|\frac{\partial G_{1}(t, s)}{\partial t}\right| d s & \\
\leq & \frac{T^{\alpha-1}}{\Gamma(\alpha)}\left(\frac{3}{2}+\frac{\alpha^{3}-3 \alpha^{2}+14 \alpha-12}{48}\right)=U_{3}, \\
t \in[0, T], & t \in \\
\int_{0}^{T}\left|\frac{\partial G_{2}(t, s)}{\partial t}\right| d s & =\frac{T^{\beta-1}}{\Gamma(\beta)}\left(\frac{3}{2}+\frac{\beta^{3}-3 \beta^{2}+14 \beta-12}{48}\right) \\
& t \in[0, T] .
\end{aligned}
$$

Proof. For any $t \in[0, T]$,

$$
\begin{aligned}
\int_{0}^{T} \mid G_{1}( & t, s) \mid d s \\
\leq & \left|\int_{0}^{t} \frac{(t-s)^{\alpha-1}}{\Gamma(\alpha)} d s\right|+\frac{1}{2}\left|\int_{0}^{T} \frac{(T-s)^{\alpha-1}}{\Gamma(\alpha)} d s\right| \\
& +\frac{|T-2 t|}{4} \int_{0}^{T} \frac{(T-s)^{\alpha-2}}{\Gamma(\alpha-1)} d s \\
& +\frac{|t(T-t)|}{4} \int_{0}^{T} \frac{(T-s)^{\alpha-3}}{\Gamma(\alpha-2)} d s \\
& +\frac{\left|6 T t^{2}-4 t^{3}-T^{3}\right|}{48} \int_{0}^{T} \frac{(T-s)^{\alpha-4}}{\Gamma(\alpha-3)} d s \\
& +\frac{\left|2 T t^{3}-t T^{3}-t^{4}\right|}{48} \int_{0}^{T} \frac{(T-s)^{\alpha-5}}{\Gamma(\alpha-4)} d s \\
\leq & \frac{T^{\alpha}}{\Gamma(\alpha+1)}+\frac{T^{\alpha}}{2 \Gamma(\alpha+1)}+\frac{T^{\alpha}}{4 \Gamma(\alpha)} \\
& \left.+\frac{T^{\alpha}}{16 \Gamma(\alpha-1)}+\frac{T^{\alpha}}{48 \Gamma(\alpha-2)}+\frac{5}{768 \Gamma(\alpha-3)}+\frac{5 \alpha^{4}-14 \alpha^{3}+55 \alpha^{2}+146 \alpha}{768}\right)
\end{aligned}
$$

On the other hand,

$$
\begin{gathered}
\int_{0}^{T}\left|\frac{\partial G_{1}(t, s)}{\partial t}\right| d s \\
=\int_{0}^{t} \frac{(t-s)^{\alpha-2}}{\Gamma(\alpha-1)} d s
\end{gathered}
$$

$$
\begin{gathered}
+\int_{0}^{T} \mid-\frac{(T-s)^{\alpha-2}}{2 \Gamma(\alpha-1)}+\frac{(T-2 t)(T-s)^{\alpha-3}}{4 \Gamma(\alpha-2)} \\
+\frac{\left(T t-t^{2}\right)(T-s)^{\alpha-4}}{4 \Gamma(\alpha-3)} \\
+\frac{\left(6 T t^{2}-T^{3}-4 t^{3}\right)(T-s)^{\alpha-5}}{48 \Gamma(\alpha-4)} \mid d s
\end{gathered}
$$




$$
\begin{aligned}
\leq & \frac{T^{\alpha-1}}{\Gamma(\alpha)}+\frac{T^{\alpha-1}}{2 \Gamma(\alpha)}+\frac{|T-2 t| T^{\alpha-2}}{4 \Gamma(\alpha-1)} \\
& +\frac{\left|T t-t^{2}\right| T^{\alpha-3}}{4 \Gamma(\alpha-2)}+\frac{\left|6 T t^{2}-T^{3}-4 t^{3}\right| T^{\alpha-4}}{48 \Gamma(\alpha-3)} \\
\leq & \frac{T^{\alpha-1}}{\Gamma(\alpha)}+\frac{T^{\alpha-1}}{2 \Gamma(\alpha)}+\frac{T^{\alpha-1}}{4 \Gamma(\alpha-1)} \\
& +\frac{T^{\alpha-1}}{16 \Gamma(\alpha-2)}+\frac{T^{\alpha-1}}{48 \Gamma(\alpha-3)} \\
\leq & \frac{T^{\alpha-1}}{\Gamma(\alpha)}\left(\frac{3}{2}+\frac{\alpha^{3}-3 \alpha^{2}+14 \alpha-12}{48}\right) .
\end{aligned}
$$

Inequalities (11) and (13) can be proved in the same way.

The first result is based on Leray-Schauder alternative.

Lemma 7 (see [24]). Let $F: E \rightarrow E$ be a completely continuous operator (i.e., a map that is restricted to any bounded set in $E$ is compact). Let

$$
\varepsilon(F)=\{x \in E: x=\lambda F(x) \text { for some } 0<\lambda<1\} .
$$

Then either the set $\varepsilon(F)$ is unbounded or $F$ has at least one fixed point.

Theorem 8. Let $f$ and $g$ satisfy the following growth condition:

$$
\begin{gathered}
|f(t, x, y)| \leq m_{0}+m_{1}|x|+m_{2}|y|, \\
m_{i} \geq 0 \quad(i=1,2), \quad m_{0}>0, \\
|g(t, x, y)| \leq n_{0}+n_{1}|x|+n_{2}|y|, \\
n_{i} \geq 0 \quad(i=1,2), \quad n_{0}>0 .
\end{gathered}
$$

In addition, it is assumed that

$$
U_{1} m_{1}+U_{2} n_{1}<1, \quad U_{1} m_{2}+U_{2} n_{2}<1 .
$$

Then problem (1) has at least one solution.

For sake of convenience, we set $U_{0}=\min \left\{1-\left(U_{1} m_{1}+\right.\right.$ $\left.\left.U_{2} n_{1}\right), 1-\left(U_{1} m_{2}+U_{2} n_{2}\right)\right\}$.

Proof. First, we show that operator $F: X \times X \rightarrow X \times X$ is completely continuous.

Step 1. In view of the continuity of $f, g, x(t), y(t)$ and $G_{1}(t, s)$, $G_{2}(t, s)$, it is obvious that the operator $F$ is continuous.

Step 2. Let $\Omega \subset X \times X$ be bounded; then there exist positive constants $K_{1}$ and $K_{2}$ such that

$$
\begin{aligned}
|f(t, x(t), y(t))| \leq K_{1}, \quad & |g(t, x(t), y(t))| \leq K_{2}, \\
& \forall(x, y) \in \Omega, t \in[0, T] .
\end{aligned}
$$

Then for any $(x, y) \in \Omega$, according to the inequalities (10) and (11), we have

$$
\left|F_{1}(x, y)(t)\right|=\left|\int_{0}^{T} G_{1}(t, s) f(s, x(s), y(s)) d s\right| \leq K_{1} U_{1} .
$$

Similarly, we get

$$
\left|F_{2}(x, y)(t)\right|=\left|\int_{0}^{T} G_{2}(t, s) g(s, x(s), y(s)) d s\right| \leq K_{2} U_{2} .
$$

Thus, it follows from the above inequalities that the operator $F$ is uniformly bounded.

Step 3. We show that $F$ is equicontinuous:

$$
\begin{aligned}
\left|\left(F_{1}(x, y)\right)_{t}^{\prime}(t)\right| & =\left|\int_{0}^{T} \frac{\partial G_{1}(t, s)}{\partial t} f(s, x(s), y(s)) d s\right| \\
& \leq K_{1} \int_{0}^{T}\left|\frac{\partial G_{1}(t, s)}{\partial t}\right| d s \leq K_{1} U_{3} .
\end{aligned}
$$

Hence, for $0 \leq t_{1} \leq t_{2} \leq T$, by the inequalities (12) and (13), we have

$$
\begin{aligned}
& \left|F_{1}(x, y)\left(t_{2}\right)-F_{1}(x, y)\left(t_{1}\right)\right| \\
& \quad \leq \int_{t_{1}}^{t_{2}}\left|\left(F_{1}(x, y)\right)_{s}^{\prime}(s)\right| d s \leq K_{1} U_{3}\left|t_{2}-t_{1}\right| .
\end{aligned}
$$

Analogously, we can obtain

$$
\begin{aligned}
\left|F_{2}(x, y)\left(t_{2}\right)-F_{2}(x, y)\left(t_{1}\right)\right| & \leq \int_{t_{1}}^{t_{2}}\left|\left(F_{2}(x, y)\right)_{s}^{\prime}(s)\right| d s \\
& \leq K_{2} U_{4}\left|t_{2}-t_{1}\right| .
\end{aligned}
$$

Therefore, the operator $F(x, y)$ is equicontinuous, and thus the operator $F(x, y)$ is completely continuous.

Finally, it will be verified that the set $\varepsilon=\{(x, y) \in X \times X$ : $(x, y)=\lambda F(x, y), 0 \leq \lambda \leq 1\}$ is bounded.

Let $(x, y) \in \varepsilon$, then $(x, y)=\lambda F(x, y)$. For any $t \in[0, T]$, we have $x(t)=\lambda F_{1}(x, y)(t), y(t)=\lambda F_{2}(x, y)(t)$; then,

$$
\begin{gathered}
|x(t)| \leq\left|F_{1}(x, y)(t)\right| \leq\left(m_{0}+m_{1}|x(t)|+m_{2}|y(t)|\right) U_{1}, \\
|y(t)| \leq\left(n_{0}+n_{1}|x(t)|+n_{2}|y(t)|\right) U_{2} .
\end{gathered}
$$

Hence, we have

$$
\begin{gathered}
\|x\| \leq\left(m_{0}+m_{1}\|x\|+m_{2}\|y\|\right) U_{1}, \\
\|y\| \leq\left(n_{0}+n_{1}\|x\|+n_{2}\|y\|\right) U_{2},
\end{gathered}
$$

which imply that

$$
\begin{aligned}
\|x\|+\|y\| \leq & \left(U_{1} m_{0}+U_{2} n_{0}\right)+\left(U_{1} m_{1}+U_{2} n_{1}\right)\|x\| \\
& +\left(U_{1} m_{2}+U_{2} n_{2}\right)\|y\| .
\end{aligned}
$$


As a result,

$$
\|(x, y)\| \leq \frac{U_{1} m_{0}+U_{2} n_{0}}{U_{0}},
$$

for any $t \in[0, T]$, which proves that $\varepsilon$ is bounded; thus by Lemma 7, the operator $F$ has at least one fixed point. Hence the boundary value problem (1) has at least one solution; this completes the proof.

In the second result, we prove uniqueness of solutions of the boundary value problem (1) via contraction mapping principle.

Theorem 9. Let $f$ and $g$ satisfy the following growth conditions:

$\left(H_{1}\right)$ there exist two constants $L_{i}>0$ and $\lambda_{i}>0, i=1,2$ such that

$$
\begin{array}{r}
\left|f\left(t, x_{1}, y_{1}\right)-f\left(t, x_{2}, y_{2}\right)\right| \leq L_{1}\left|x_{1}-x_{2}\right|+L_{2}\left|y_{1}-y_{2}\right| \\
\left|g\left(t, x_{1}, y_{1}\right)-g\left(t, x_{2}, y_{2}\right)\right| \leq \lambda_{1}\left|x_{1}-x_{2}\right|+\lambda_{2}\left|y_{1}-y_{2}\right| \\
t \in[0, T], x_{1}, x_{2}, y_{1}, y_{2} \in \mathbb{R}
\end{array}
$$

$\left(\mathrm{H}_{2}\right)$ Consider

$$
\max \left\{L_{1} U_{1}+\lambda_{1} U_{2}, L_{2} U_{1}+\lambda_{2} U_{2}\right\}=L<1 .
$$

Then problem (1) has a unique solution.

Proof. Let $\left(x_{1}, y_{1}\right),\left(x_{2}, y_{2}\right) \in X \times X$; then

$$
\begin{aligned}
& \left|F_{1}\left(x_{2}, y_{2}\right)(t)-F_{1}\left(x_{1}, y_{1}\right)(t)\right| \\
& =\mid \int_{0}^{T} G_{1}(t, s) f\left(s, x_{2}(s), y_{2}(s)\right) d s \\
& \quad-\int_{0}^{T} G_{1}(t, s) f\left(s, x_{1}(s), y_{1}(s)\right) d s \mid \\
& \leq \int_{0}^{T}\left|G_{1}(t, s)\right| \mid f\left(s, x_{2}(s), y_{2}(s)\right) \\
& \quad-f\left(s, x_{1}(s), y_{1}(s)\right) \mid d s \\
& \leq U_{1}\left(L_{1}\left\|x_{2}-x_{1}\right\|+L_{2}\left\|y_{2}-y_{1}\right\|\right) .
\end{aligned}
$$

Analogously,

$$
\begin{aligned}
& \left|F_{2}\left(x_{2}, y_{2}\right)(t)-F_{2}\left(x_{1}, y_{1}\right)(t)\right| \\
& \quad \leq U_{2}\left(\lambda_{1}\left\|x_{2}-x_{1}\right\|+\lambda_{2}\left\|y_{2}-y_{1}\right\|\right) .
\end{aligned}
$$

Thus,

$$
\begin{aligned}
&\left\|F\left(x_{2}, y_{2}\right)-F\left(x_{1}, y_{1}\right)\right\| \\
&=\left\|F_{1}\left(x_{2}, y_{2}\right)-F_{1}\left(x_{1}, y_{1}\right)\right\| \\
& \quad+\left\|F_{2}\left(x_{2}, y_{2}\right)-F_{2}\left(x_{1}, y_{1}\right)\right\|
\end{aligned}
$$

$$
\begin{aligned}
\leq & \left(L_{1} U_{1}+\lambda_{1} U_{2}\right)\left\|x_{2}-x_{1}\right\| \\
& +\left(L_{2} U_{1}+\lambda_{2} U_{2}\right)\left\|y_{2}-y_{1}\right\| \\
\leq & L\left(\left\|x_{2}-x_{1}\right\|+\left\|y_{2}-y_{1}\right\|\right) \\
= & L\left\|\left(x_{2}, y_{2}\right)-\left(x_{1}, y_{1}\right)\right\| .
\end{aligned}
$$

Hence, we conclude that problem (1) has a unique solution by $\left(\mathrm{H}_{2}\right)$ and the contraction mapping principle; this ends the proof.

\section{Examples}

In this section, two examples are given in order to verify the validity of Theorems 8 and 9 .

Example 1. Consider the system

$$
\begin{gathered}
{ }^{c} D^{17 / 4} x(t)+\frac{1}{2}(t+x(t)+y(t))=0, \quad 0<t<1, \\
{ }^{c} D^{9 / 2} y(t)+\frac{3}{2}(\sqrt{t}+x(t)+y(t))=0, \quad 0<t<1, \\
x^{(i)}(0)=-x^{(i)}(1), \quad i=0,1,2,3,4, \\
y^{(i)}(0)=-y^{(i)}(1), \quad i=0,1,2,3,4,
\end{gathered}
$$

where $T=1,|f(t, x, y)| \leq 1+|x|+|y|,|g(t, x, y)| \leq 2+2|x|+$ $2|y|, \alpha=17 / 4, \beta=9 / 2, m_{0}=m_{1}=m_{2}=1, n_{0}=n_{1}=n_{2}=2$. Consider

$$
\begin{aligned}
& U_{1}=\frac{T^{\alpha}}{\Gamma(\alpha+1)}\left(\frac{3}{2}+\frac{5 \alpha^{4}-14 \alpha^{3}+55 \alpha^{2}+146 \alpha}{768}\right) \approx 0.1229 \\
& U_{2}=\frac{T^{\beta}}{\Gamma(\beta+1)}\left(\frac{3}{2}+\frac{5 \beta^{4}-14 \beta^{3}+55 \beta^{2}+146 \beta}{768}\right) \approx 0.0920 .
\end{aligned}
$$

Then $U_{1} m_{1}+U_{2} n_{1}<1$ and $U_{1} m_{2}+U_{2} n_{2}<1$; by Theorem 8 , the existence of the solution for the system (34) is obvious.

Example 2. Consider the system

$$
\begin{gathered}
{ }^{c} D^{17 / 4} x(t)+t+\sin x(t)+2 \frac{|y(t)|}{1+|y(t)|}=0, \quad 0<t<1, \\
{ }^{c} D^{9 / 2} y(t)+t^{2}+2 \frac{|x(t)|}{1+|x(t)|}+\arctan y(t)=0, \quad 0<t<1, \\
x^{(i)}(0)=-x^{(i)}(1), \quad i=0,1,2,3,4, \\
y^{(i)}(0)=-y^{(i)}(1), \quad i=0,1,2,3,4,
\end{gathered}
$$

where $T=1, f(t, x, y)=t+\sin x(t)+2(|y(t)| /(1+|y(t)|))$, $g(t, x, y)=t^{2}+2(|x(t)| /(1+|x(t)|))+\arctan y(t) \cdot \alpha=17 / 4$, $\beta=9 / 2$. 
Noting that

$$
\left|(\sin x)^{\prime}\right|=|\cos x| \leq 1, \quad\left|(\arctan y)^{\prime}\right|=\frac{1}{1+y^{2}} \leq 1,
$$

we have

$$
\begin{aligned}
& \left|f\left(t, x_{2}, y_{2}\right)-f\left(t, x_{1}, y_{1}\right)\right| \\
& \quad=\left|\sin x_{2}(t)-\sin x_{1}(t)\right|+2\left|\frac{\left|y_{2}(t)\right|}{1+\left|y_{2}(t)\right|}\right|-\left|\frac{\left|y_{1}(t)\right|}{1+\left|y_{1}(t)\right|}\right| \\
& \quad \leq\left\|x_{2}-x_{1}\right\|+2\left\|y_{2}-y_{1}\right\|, \\
& \quad\left|g\left(t, x_{2}, y_{2}\right)-g\left(t, x_{1}, y_{1}\right)\right| \leq 2\left\|x_{2}-x_{1}\right\|+\left\|y_{2}-y_{1}\right\|, \\
& U_{1}=\frac{T^{\alpha}}{\Gamma(\alpha+1)}\left(\frac{3}{2}+\frac{5 \alpha^{4}-14 \alpha^{3}+55 \alpha^{2}+146 \alpha}{768}\right) \approx 0.1229 \\
& U_{2}=\frac{T^{\beta}}{\Gamma(\beta+1)}\left(\frac{3}{2}+\frac{5 \beta^{4}-14 \beta^{3}+55 \beta^{2}+146 \beta}{768}\right) \approx 0.0920 .
\end{aligned}
$$

Obviously,

$$
\max \left\{L_{1} U_{1}+\lambda_{1} U_{2}, L_{2} U_{1}+\lambda_{2} U_{2}\right\}<1
$$

then we can conclude from Theorem 9 that system (36) has a unique solution.

\section{Conflict of Interests}

The authors declare that there is no conflict of interests regarding the publication of this paper.

\section{Acknowledgments}

The authors express their thanks to the referee for his/her valuable suggestions. The second author was supported by NSF of China (11271154) and the first author was supported by the Fundamental Research Funds for the Central Universities (13cx02015A).

\section{References}

[1] S. G. Samko, A. A. Kilbas, and O. I. Marichev, Fractional Integrals and Derivatives: Theory and Applications, Gordon and Breach Science Publishers, Yverdon, Switzerland, 1993.

[2] I. Podlubny, Fractional Differential Equations, Academic Press, San Diego, Calif, USA, 1999.

[3] A. A. Kilbas, H. M. Srivastava, and J. J. Trujillo, Theory and Applications of Fractional Differential Equations, vol. 204 of North-Holland Mathematics Studies, Elsevier Science, Amsterdam, The Netherlands, 2006.

[4] J. Sabatier, O. P. Agrawal, and J. A. T. Machado, Eds., Advances in Fractional Calculus: Theoretical Developments and Applications in Physics and Engineering, Springer, Dordrecht, The Netherlands, 2007.
[5] A. Alsaedi, B. Ahmad, and A. Assolami, "On antiperiodic boundary value problems for higher-order fractional differential equations," Abstract and Applied Analysis, vol. 2012, Article ID 325984, 15 pages, 2012.

[6] Y.-K. Chang and J. J. Nieto, "Some new existence results for fractional differential inclusions with boundary conditions," Mathematical and Computer Modelling, vol. 49, no. 3-4, pp. 605609, 2009.

[7] Z. Bai, W. Sun, and W. Zhang, "Positive solutions for boundary value problems of singular fractional differential equations," Abstract and Applied Analysis, vol. 2013, Article ID 129640, 7 pages, 2013.

[8] Z. Bai and H. Lü, "Positive solutions for boundary value problem of nonlinear fractional differential equation," Journal of Mathematical Analysis and Applications, vol. 311, no. 2, pp. 495-505, 2005.

[9] R. P. Agarwal, M. Benchohra, and S. Hamani, "A survey on existence results for boundary value problems of nonlinear fractional differential equations and inclusions," Acta Applicandae Mathematicae, vol. 109, no. 3, pp. 973-1033, 2010.

[10] R. P. Agarwal, M. Belmekki, and M. Benchohra, "A survey on semilinear differential equations and inclusions involving Riemann-Liouville fractional derivative," Advances in Difference Equations, vol. 2009, Article ID 918728, 47 pages, 2009.

[11] X. Xu, D. Jiang, and C. Yuan, "Multiple positive solutions to singular positone and semipositone Dirichlet-type boundary value problems of nonlinear fractional differential equations," Nonlinear Analysis: Theory, Methods \& Applications, vol. 74, no. 16, pp. 5685-5696, 2011.

[12] C.-Z. Bai and J.-X. Fang, “The existence of a positive solution for a singular coupled system of nonlinear fractional differential equations," Applied Mathematics and Computation, vol. 150, no. 3, pp. 611-621, 2004.

[13] X. Xu, D. Jiang, and C. Yuan, "Multiple positive solutions for the boundary value problem of a nonlinear fractional differential equation," Nonlinear Analysis: Theory, Methods \& Applications, vol. 71, no. 10, pp. 4676-4688, 2009.

[14] G. Wang, B. Ahmad, and L. Zhang, "Existence results for nonlinear fractional differential equations with closed boundary conditions and impulses," Advances in Difference Equations, vol. 2012, article 169, 2012.

[15] V. Gafiychuk, B. Datsko, V. Meleshko, and D. Blackmore, "Analysis of the solutions of coupled nonlinear fractional reactiondiffusion equations," Chaos, Solitons \& Fractals, vol. 41, no. 3, pp. 1095-1104, 2009.

[16] Y. Chen, D. Chen, and Z. Lv, “The existence results for a coupled system of nonlinear fractional differential equations with multi-point boundary conditions," Bulletin of the Iranian Mathematical Society, vol. 38, no. 3, pp. 607-624, 2012.

[17] B. Ahmad and J. J. Nieto, "Existence results for a coupled system of nonlinear fractional differential equations with threepoint boundary conditions," Computers and Mathematics with Applications, vol. 58, no. 9, pp. 1838-1843, 2009.

[18] B. Ahmad and V. Otero-Espinar, "Existence of solutions for fractional differential inclusions with antiperiodic boundary conditions," Boundary Value Problems, vol. 2009, Article ID 625347, 2009.

[19] R. P. Agarwal and B. Ahmad, "Existence theory for anti-periodic boundary value problems of fractional differential equations and inclusions," Computers and Mathematics with Applications, vol. 62 , no. 3, pp. 1200-1214, 2011. 
[20] B. Ahmad, "Existence of solutions for fractional differential equations of order $q \in(2,3]$ with anti-periodic boundary conditions," Journal of Applied Mathematics and Computing, vol. 34, no. 1-2, pp. 385-391, 2010.

[21] R. P. Agarwal and B. Ahmad, "Existence of solutions for impulsive anti-periodic boundary value problems of fractional semilinear evolution equations," Dynamics of Continuous, Discrete and Impulsive Systems A, vol. 18, no. 4, pp. 457-470, 2011.

[22] G. Wang, B. Ahmad, and L. Zhang, "Impulsive anti-periodic boundary value problem for nonlinear differential equations of fractional order," Nonlinear Analysis: Theory, Methods \& Applications, vol. 74, no. 3, pp. 792-804, 2011.

[23] S. K. Ntouyas and M. Obaid, "A coupled system of fractional differential equations with nonlocal integral boundary conditions," Advances in Difference Equations, vol. 2012, article 130, 2012.

[24] A. Granas and J. Dugundji, Fixed Point Theory, Springer, New York, NY, USA, 2003. 


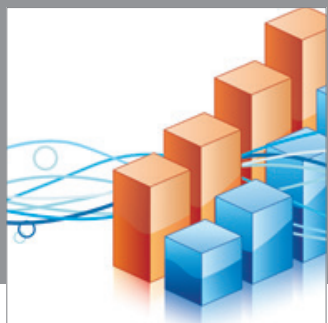

Advances in

Operations Research

mansans

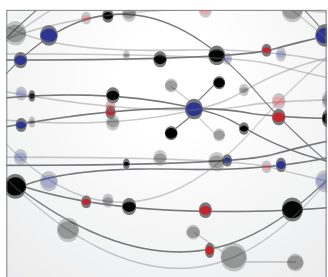

The Scientific World Journal
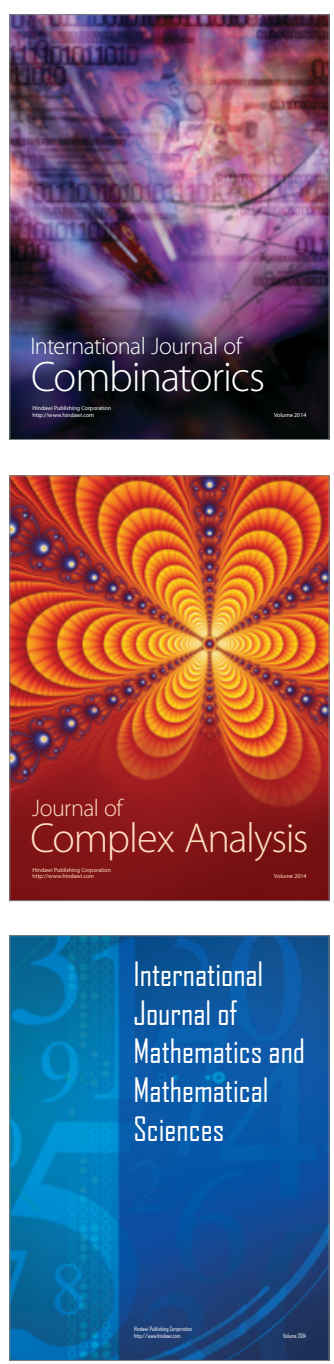
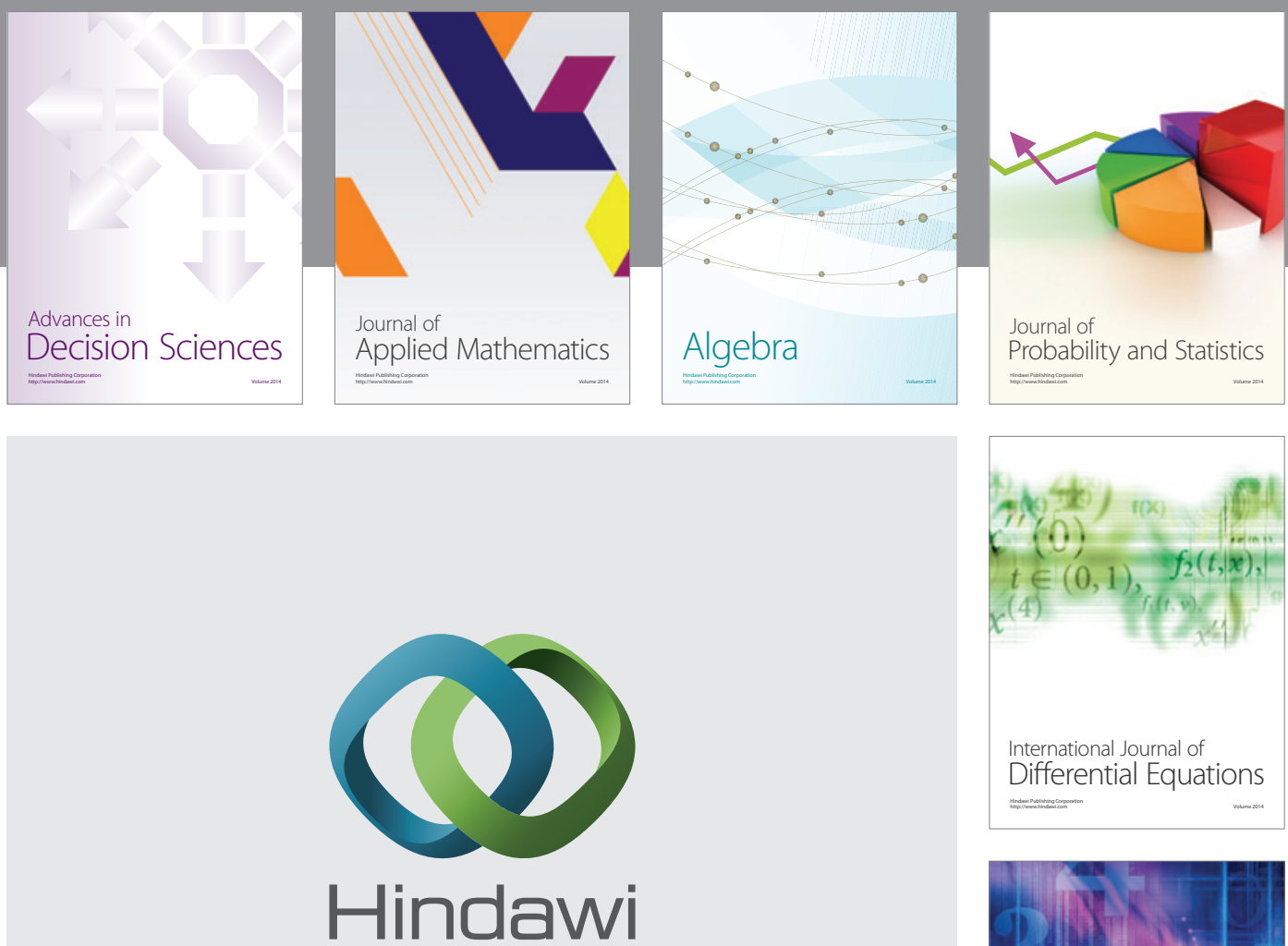

Submit your manuscripts at http://www.hindawi.com
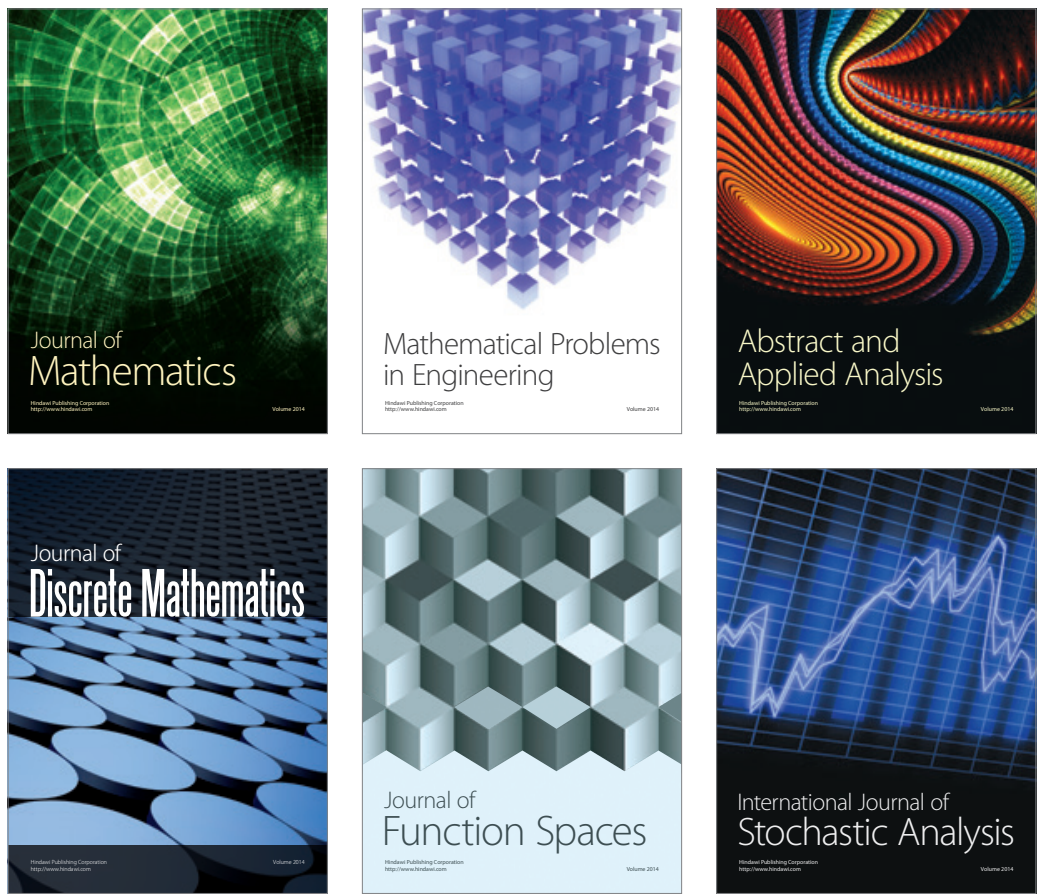

Journal of

Function Spaces

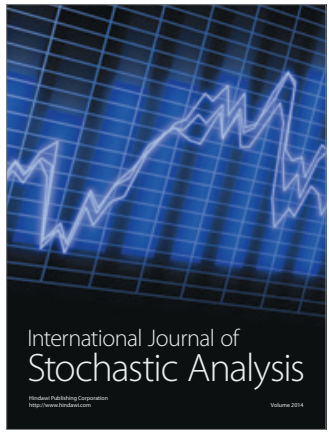

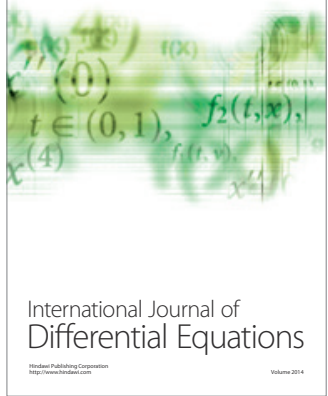
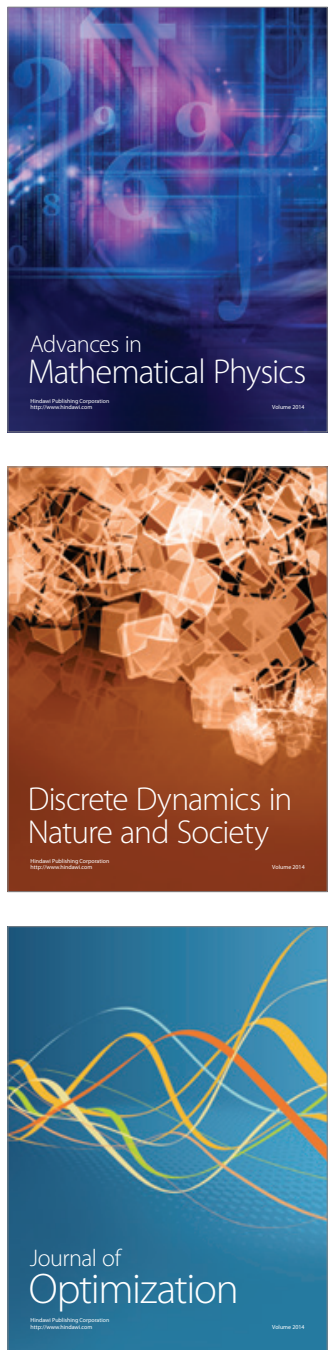